01

\title{
Модифицированный метод фарадеевского вращения для исследования атомных линий рубидия и калия В сверхтонких ячейках
}

\author{
(C) А. Саргсян ${ }^{1}$, А. Амирян ${ }^{1,2}$, Т.А. Вартанян ${ }^{3, \text {, Д. Саркисян }}{ }^{1}$ \\ ${ }^{1}$ Институт фризических исследований НАН Армении, \\ 0203 Аштарак, Армения \\ ${ }^{2}$ Laboratoire Interdisciplinaire Carnot de Bourgogne, Université Bourgogne-Franche-Comté \\ BP 47870, 21078 Dijon Cedex, France \\ ${ }^{3}$ Университет ИТМО, \\ 197101 Санкт-Петербург, Россия \\ ฯ e-mail: tigran.vartanyan@mail.ru
}

Поступила в редакцию 24.09.2018 г.

В окончательной редакции 24.09.2018 г.

Принята к публикации 30.10.2018 г.

Разработан модифицированный метод фарадеевского вращения с использованием наноячейки, заполненной атомарными парами рубидия и калия. Формируемые атомные линии имеют спектральную ширину в $1.5-2$ раза уже, чем полученные традиционным методом фарадеевского вращения в наноячейках. Новый метод позволяет формировать спектральную ширину атомной линии в 8 раз уже допплеровского уширения в случае $D_{2}$-линии рубидия и в 15 уже допплеровского уширения в случае $D_{1,2}$-линий калия. В магнитных полях $B=100-1200 \mathrm{G}$ все атомные линии атомов $\mathrm{Rb}$ и $\mathrm{K}$ спектрально разрешены и идентифицированы. В случае $D_{2}$-линии $\mathrm{Rb}$ показано, что при определенных величинах $B$ вероятности магнито-индуцированных переходов $\left({ }^{87} \mathrm{Rb}, F_{g}=1 \rightarrow F_{e}=3\right.$ и $\left.{ }^{85} \mathrm{Rb}, F_{g}=2 \rightarrow F_{e}=4\right)$ могут превосходить вероятности разрешенных переходов. Показано, что модифицированный метод фарадеевского вращения является удобным и эффективным для спектроскопии высокого разрешения.

DOI: $10.21883 / O S .2019 .03 .47362 .284-18$

\section{Введение}

Эффект вращения плоскости линейно поляризованного излучения при прохождении через резонансную среду, к которой приложено магнитное поле (фарадеевское вращение, ФВ), продолжает активно исследоваться, что обусловлено технической простотой его реализации и большим числом возможных применений [1-3]. Поскольку атомы шелочных металлов имеют простую электронную структуры (всего один валентный электрон) и сильные атомные переходы в видимой и ближней инфракрасных областях (в этих областях доступны лазеры с хорошими параметрами), они широко используются в различных областях, таких как метрология, магнитометрия, телекоммуникации и др. [1]. Для этих целей, как правило, используются заполненные парами атомов щелочных металлов спектроскопические ячейки сантиметровой длины [3].

Большое число работ посвящено созданию узкополосных (полоса пропускания $0.5-1 \mathrm{GHz}$ ) оптических атомных фильтров (ОАФ) на основе фарадеевского вращения в парах щелочных металлов на $D_{1,2}$-линиях, при этом прикладываются продольные магнитные поля $B \sim 100 \mathrm{G}$. Для ОАФ используется конфигурация со скрещенными поляризаторами и пропускается только полезный сигнал [4-7]. Ширина спектра пропускания ОАФ может быть на три порядка уже, чем у ши- роко распространенных коммерческих интерференционных фильтров. Основными элементами для ОАФ являются спектроскопические ячейки с длиной $1-100 \mathrm{~mm}$, заполненные парами щелочных металлов. Такие ячейки успешно применяются и для определения слабых магнитных полей $(B \sim 1 \mu \mathrm{G})$ по измерению величины угла ФВ в условиях, когда частота лазера близка к частоте атомного перехода [1,2]. Важно заметить, что при комнатной температуре атомные линии в спектрах пропускания в обычно используемых сантиметровых ячейках имеют допплеровски уширенный профиль, описываемый гауссовой кривой. В результате атомные линии (переходы) в регистрируемых спектрах оказываются спектрально перекрытыми, что значительно затрудняет изучение их индивидуального поведения. Ситуация особенно усложняется в случае приложения магнитных полей 500-1000 G. В этом случае атомные переходы сильно смещаются и расщепляются на большое количество новых атомных переходов. При этом, как правило, происходит значительное изменение (уменьшение или возрастание) вероятностей атомных переходов. Поэтому возможность регистрации отдельных атомных переходов очень важна для их изучения. В недавних работах [8-10] было продемонстрировано, что спектр сигнала фарадеевского вращения при прохождении резонансного излучения через наноячейку, заполненную парами атомов цезия и рубидия, и имеющую толщину $L=\lambda / 2$, где 
$\lambda$ - длина волны излучения, резонансного с частотой атомного перехода, демонстрирует десятикратное сужение по сравнению с аналогичным спектром $Ф \mathrm{~B}$, формируемым в сантиметровых ячейках. В случае использования $D_{1}$-линии $\mathrm{Cs}$ длина волны лазерного излучения составляла $\lambda=895 \mathrm{~nm}$, а в случае использования $D_{1}$-линии $\mathrm{Rb}-795 \mathrm{~nm}$. Важно отметить, что сигнал ФВ уверенно регистрируется даже при малых магнитных полях $B \sim 0.5 \mathrm{G}$, что обусловливает его практические применения. В работе [11] было продемонстрировано, что применение процесса селективного отражения (SR) от границы атомарных паров и сапфирового окна наноячейки при толщине $L \sim \lambda / 2$ также позволяет спектральное разделение большого количества атомных переходов, которые формируются в сильных магнитных полях. Некоторые особенности SR в тонких атомарных слоях приведены также в [12].

Несмотря на ряд ранее разработнных методов, которые позволяют формировать спектрально узкие, свободные от допплеровского уширения атомные переходы, работы в этом направлении продолжаются. При этом акцент делается на формирование все более узких атомных переходов при сохранении простоты метода. В настоящей работе впервые изложен метод, который является новой модификацией метода фарадеевского вращения (MFR - modification of Faraday rotation) с использованием наноячейки и позволяет формировать атомные переходы, которые в 1.5-2 раза спектрально уже, чем получаемые по обычному методу фарадеевского вращения в наноячейках. Продемонстрировано, что метод $M F R$ особенно успешно работает в случае наноячейки, заполненной парами атомов калия, поскольку в магнитном поле переходы атома $\mathrm{K}$ расщепляются на несколько атомных переходов с малым частотным расстоянием между ними $\sim 100 \mathrm{MHz}$, в то время как допплеровское уширение атомных линий $\sim 900 \mathrm{MHz}$. Тем не менее, метод MFR позволяет осуществить их уверенное спектральное разрешение.

\section{Эксперимент}

\section{Конструкция наноячеек}

Фотография наноячейки (НЯ), заполненной естественной смесью изотопов рубидия (72\% изотопа ${ }^{85} \mathrm{Rb}$ и $28 \%$ изотопа $\left.{ }^{87} \mathrm{Rb}\right)$, приведена в работе [9]. Окна ячейки изготовлены из хорошо отполированного кристаллического сапфира с размерами $20 \times 30 \mathrm{~mm}$ и толщиной $1.2 \mathrm{~mm}$. Для обеспечения клиновидности зазора между окнами (в нижней части кюветы) до склейки помещались две платиновые пластинки толщиной $1 \mu \mathrm{m}$ и размерами $1 \times 1 \mathrm{~mm}$. Для минимизации двулучепреломления окна изготовлены так, чтобы ось $C$ была перпендикулярна поверхности окна. Область $L=\lambda / 2=397.5 \mathrm{~nm}$ отмечена овалом на рис. 1 в работе [9]. В эксперименте температура отростка наноячейки составляла $120^{\circ} \mathrm{C}$, что обеспечивало концентрацию атомов $N=2 \cdot 10^{13} \mathrm{~cm}^{-3}$.
Фотография НЯ, заполненной калием (93.25\% изотопа ${ }^{39} \mathrm{~K}, 0.01 \%$ изотопа ${ }^{40} \mathrm{~K}$ и $6.7 \%$ изотопа $\left.{ }^{41} \mathrm{~K}\right)$, приведена в работах [13-15]. Конструкция НЯ аналогична конструкции наноячейки, заполненной рубидием. В эксперименте температура отростка наноячейки с $\mathrm{K}$ составляла $160^{\circ} \mathrm{C}$, что обеспечивало концентрацию атомов $N \sim 10^{13} \mathrm{~cm}^{-3}$.

\section{Экспериментальная установка}

На рис. 1 приведена экспериментальная схема для регистрации спектра сигнала ФВ при использовании наноячейки (2), заполненной либо $\mathrm{Rb}$, либо K. Использовалось непрерывное узкополосное излучение диодного лазера с внешним резонатором (extended cavity diode laser, $E C D L$ ) с $\lambda=780 \mathrm{~nm}$ (в случае $\mathrm{Rb}, D_{2}$-линии), а также с длинами волн $\lambda=770$ и $767 \mathrm{~nm}\left(\mathrm{~K}, D_{1}\right.$ - и $D_{2}$-линии соответственно). Спектральная ширина линии лазеров $\sim 1 \mathrm{MHz}$. Для формирования больших магнитных полей использовался откалиброванный с помощью магнитометра Teslameter HT201 сильный постоянный магнит $(P M)$ из сплава неодим-железо-бор, который помещался вблизи выходного окна наноячейки НЯ. Для формирования частотного репера (ЧР), часть лазерного излучения направлялась на дополнительную сантиметровую ячейку, формирующую спектр „насыщенного поглощения“, который являлся частотным репером (Ref) для регистрируемого спектра [16]. Для формирования линейно поляризованного излучения использовался поляризатор Глана-Тейлора $\left(G_{1}\right)$, а спектры сигналов ФВ регистрировались на выходе из второго (выходного) скрещенного поляризатора $G_{2}$ (анализатора). Регистрация излучений проводилась фотодиодами ФД-24К (3). Сигналы с фотодиодов усиливались операционным усилителем и подавались на 4-лучевой цифровой осциллограф Tektronix TDS2014B или на цифровой осциллограф Siglent. Для селекции сигнала ФВ использовался интерференционный фильтр IF с шириной пропускания на полувысоте $10 \mathrm{~nm}$. Для формирования пучка диаметром $1 \mathrm{~mm}$, равным размеру области паров с толщиной

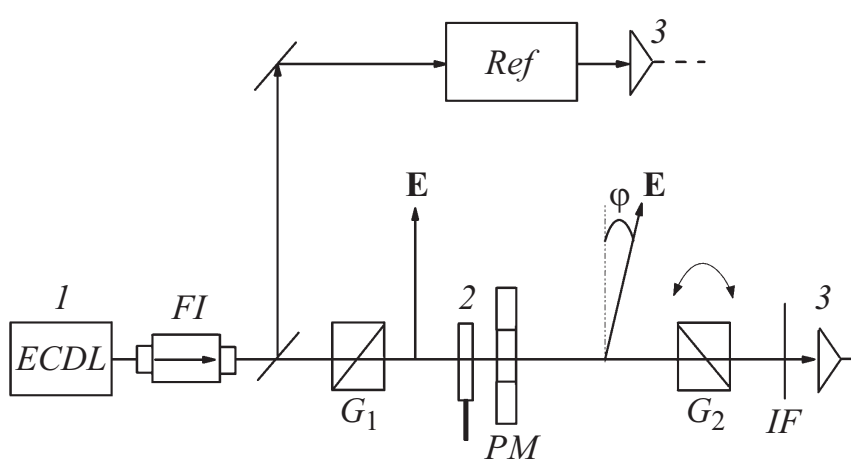

Рис. 1. Схема экспериментальной установки. $E C D L-$ диодный лазер; $F I-$ фарадеевский изолятор; $G_{1,2}-$ поляризаторы, 2 - наноячейка с $\mathrm{Rb}$ или $\mathrm{K} ; 3-$ фотоприемники, $\varphi-$ угол вращения плоскости поляризации, $P M-$ сильный постоянный магнит, $I F-$ интерференционный фильтр, Ref - узел для формирования частотного репера. 


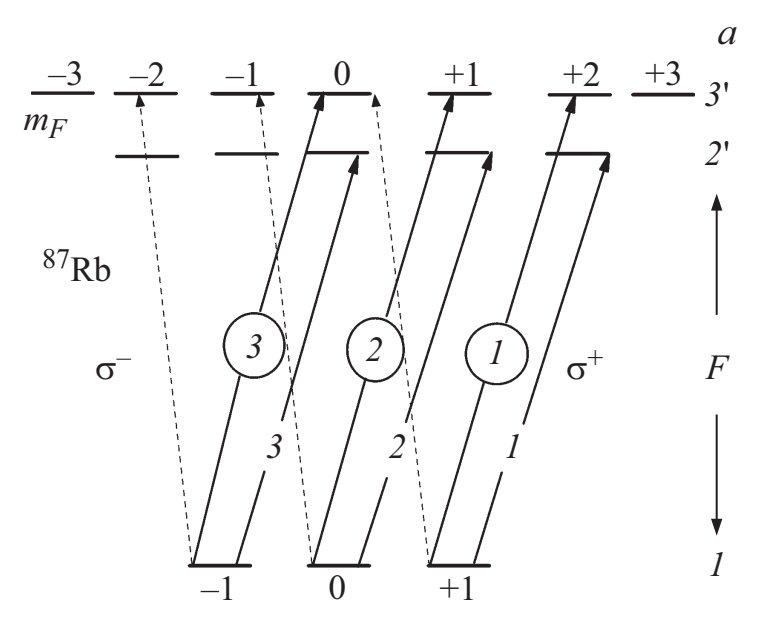

$b$

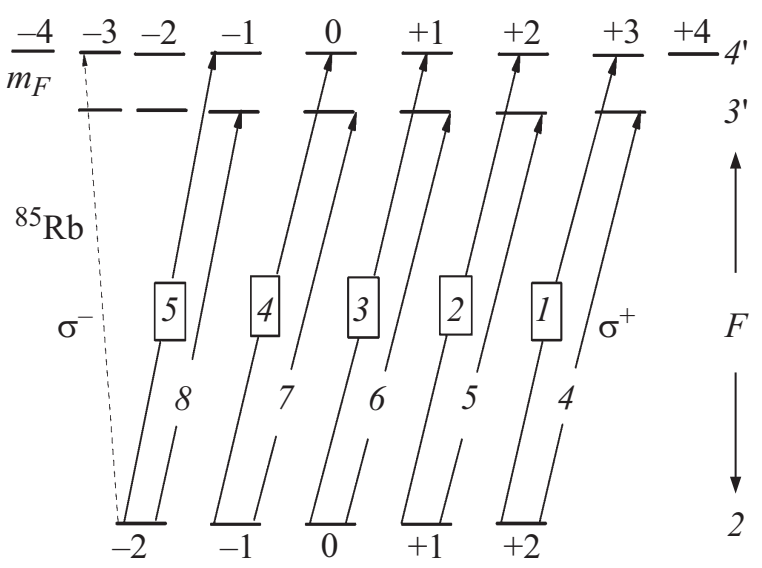

Pис. 2. (a) диаграмма переходов ${ }^{87} \mathrm{Rb}, D_{2}, 1-3-$ переходы в кружках запрещены при $B=0$, однако в магнитном поле происходит гигантское возрастание их вероятностей, показаны переходы с круговой поляризяцией $\sigma^{+}$и $\sigma^{-}$(пунктир), (b) диаграмма переходов ${ }^{85} \mathrm{Rb}, D_{2}, 1-5-$ переходы в прямоугольниках запрещены при $B=0$, однако в магнитном поле происходит гигантское возрастание их вероятностей, показаны переходы с поляризацией $\sigma^{+}$и $\sigma^{-}$(пунктир).

$L=\lambda / 2$ (рис. 1), использовалась диафрагма (на схеме не показана).

\section{Экспериментальные результаты и обсуждение}

\section{Пары атомов $\mathbf{R b}$}

На рис. 2 показаны диаграммы атомных переходов ${ }^{87} \mathrm{Rb}(a)$ и ${ }^{85} \mathrm{Rb}(b)$ в сильных магнитных полях, которые регистрируются в эксперименте. По правилам отбора для разрешенных в дипольном приближении переходов между нижними и верхними уровнями сверхтонкой структуры для полного момента атома $F$ в нулевом магнитном поле должны выполняться правила отбора $F_{e}-F_{g}=\Delta F=0, \pm 1$, а для магнитных подуровней $m_{F^{\prime}}-m_{F}= \pm 1$, для излучения с круговой поляризацией $\sigma^{+}$(левой) или $\sigma^{-}$(правой). Известно, что в сильных магнитных полях может происходить значительное возрастание вероятности (интенсивности) атомных переходов с правилами отбора $F_{e}-F_{g}= \pm 2$ [3,17-19]. Как показано ниже, при определенных значениях магнитного поля вероятности этих переходов, которые запрещены в нулевом магнитном поле, могут превосходить вероятности разрешенных атомных переходов. Поэтому мы назвали их магнито-индуцированные $(M I)$ переходы $[20,21]$. На диаграмме на рис. 2 для атомов ${ }^{87} \mathrm{Rb}$ магнито-индуцированные переходы отмечены в кружках, а для атомов ${ }^{85} \mathrm{Rb}$ в прямоугольниках. Заметим, что в процессе фарадеевского вращения участвует излучение с круговой поляризацией как $\sigma^{+}$, так и $\sigma^{-}$. Однако, чтобы сильно не усложнять диаграммы, для ${ }^{87} \mathrm{Rb}$ на рис. 2, а для $M I$-переходов показаны переходы для обеих поляризаций (для $\sigma^{-}$переходы показаны пунктиром), a для ${ }^{85} \mathrm{Rb}$ для круговой поляризации $\sigma^{-}$пунктиром показан только один переход.

Для регистрации спектра сигнала фарадеевского вращения использовалась отмеченная выше схема со скрещенными поляризаторами с приложением продольного магнитного поля [8-10]. Наноячейка толщиной $L=\lambda / 2=390 \mathrm{~nm}$ содержала пары атомов $\mathrm{Rb}$, и к ней прикладывалось продольное магнитное поле $B=600 \mathrm{G}$. Выходной поляризатор Глана (анализатор) помещался в механический держатель, который позволял осуществлять его плавное вращение вокруг оси, как по часовой стрелке, так и против часовой стрелки. Угол, на который поворачивался анализатор относительно скрещенного положения, составлял $0.1 \mathrm{rad}$. При этом на выходе анализатора регистрируется дисперсионный спектр, который на рисунке показан кривой MFR. Используемый осциллограф Siglent SDS1202x позволяет в реальном времени формировать производную кривой $M F R$, представленную на рисунке кривой $D M F R$ (derivative MFR). Магнито-индуцированные переходы $1-3$ в кружках - это переходы ${ }^{87} \mathrm{Rb}, F_{g}=1 \rightarrow F_{e}=3$, а $M I$-переходы 1-4 в прямоугольниках - это переходы ${ }^{85} \mathrm{Rb}, F_{g}=2 \rightarrow F_{e}=4$. Из сравнения кривых $F R$ и $D M F R$ видно, что формируемые атомные линии в случае $D M F R$ имеют спектральную ширину в $1.5-2$ раза уже. Это позволяет избегать перекрытия спектров атомных линий, что важно для правильной идентификации их частоты и амплитуды. К примеру, переходы 1 и 3 (в кружке), а также 1 в прямоугольнике и переход 3 хорошо разрешены в спектре $D M F R$, однако сильно перекрыты в спектре $F R$. Нижняя кривая - частотный репер при $B=0$. На pис. 3, $a$ приведены экспериментальные спектры сигналов $F R, M F R$, а также $D M F R$ (производная от $M F R$, полученная с помощью осциллографа Siglent) при толщине $L=\lambda / 2=390 \mathrm{~nm}$ и магнитном поле $B=600 \mathrm{G}$. На рис. $3, b$ приведен экспериментальный спектр сигнала поглощения $(A b s)$ при толщине $L=\lambda / 2=390$ nт и магнитном поле $B=750 \mathrm{G}$. Кривая $M F R$ показывает спектр сигнала на выходе из анализатора, который повернут на $0.1 \mathrm{rad}$. На рис. $3, b$ кривой $D M F R$ показана производная 

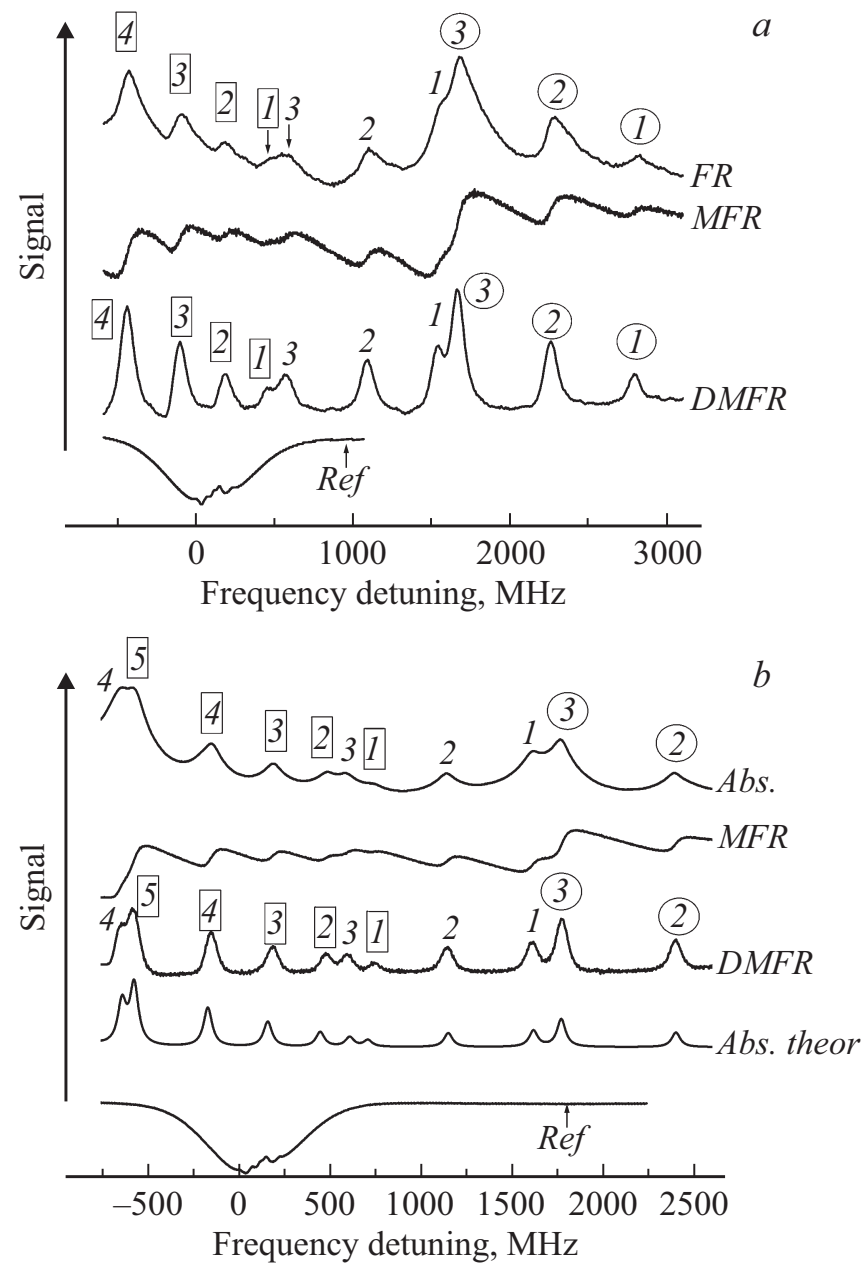

Рис. 3. (а) Верхняя кривая - спектр сигнала $F R, B=600 \mathrm{G}$, кривая $M F R$ - спектр сигнала модифицированного $F R$, кривая $D M F R$ - спектр сигнала производной кривой $M F R ; 1-3$ в кружках и 1-4 в прямоугольниках - MI-переходы атомов ${ }^{87} \mathrm{Rb}$ и ${ }^{85} \mathrm{Rb}$ соответственно. Обозначение переходов приведено на pис. 2. Нижняя кривая $R e f-$ частотный репер; $(b)$ Верхняя кривая - спектр поглощения НЯ, $B=750 \mathrm{G}$, кривая $M F R-$ спектр сигнала модифицированного $F R$, кривая $D M F R-$ спектр сигнала производной кривой $M F R, 1-3$ в кружках и $1-5$ в прямоугольниках показаны $M I$-переходы атомов ${ }^{87} \mathrm{Rb}$ и ${ }^{85} \mathrm{Rb}$ соответственно, обозначение переходов приведено на рис. 2, кривая Abs. theor - расчетный спектр поглощения НЯ, нижняя кривая - частотный репер.

кривой MFR. Кривая Abs. theor показывает расчетный спектр поглощения: спектр показывает точное частотное положение атомных линий и их амплитуды. Из сравнения кривых $A b s$ и $D M F R$ видно, что формируемые атомные линии в случае $D M F R$ (ширина $70 \mathrm{MHz}$ ) имеют спектральную ширину в 2-2.5 раза уже, поэтому все одинадцать атомных линий хорошо разрешены, в то время как в спектре, показанном кривой $A b s$, наблюдается сильное перекрытие ряда атомных линий.

По рис. $3, b$ видно, что теоретическая кривая хорошо описывает частотное положение атомных линий и их амплитуды, полученные методом DMFR. Нижняя кривая Ref - частотный репер. На рис. 4 приведен измеренный в эксперименте спектр сигнала поглощения (Abs. exp) при толщине $L=\lambda / 2=390 \mathrm{~nm}$ и магнитном поле $B=1150 \mathrm{G}$. Расчетный спектр сигнала поглощения (Abs. exp) при $L=\lambda / 2=390 \mathrm{~nm}$ показан кривой (Abs.theory). Кривая DMFR показывает производную спектра сигнала $M F R$ на выходе из анализатора, который повернут на $0.1 \mathrm{mrad}$. Из сравнения кривых $A b s . \exp$ и $D M F R$ видно, что формируемые атомные линии при методе DMFR имеют малую спектральную ширину, благодаря чему все пятнадцать атомных линий хорошо разрешены. По рис. 4 видно, что теоретическая кривая хорошо описывает частотное положение атомных линий и их амплитуды, полученные методом $D M F R$. Нижняя кривая - частотный репер при нулевом магнитном поле. Отметим, что на рис. 3-5 приведено высокочастотное крыло спектра, возбуждаемое излучением $\sigma^{+}$-поляризацией, аналогичный спектр формируется и на низкочастотном крыле при использовании излучения с $\sigma^{-}$-поляризацией. Как видно из рис. 3 и 4, при всех приложенных магнитных полях $M I$ переход под номером 3 в кружке имеет максимальную амплитуду для переходов ${ }^{87} \mathrm{Rb}$, которые начинаются с нижнего уровня $F_{g}=1$ (см. диаграмму на рис. $2, a$ ). Также относительно большие амплитуды имеют $M I$-переходы с номерами 2 и 3 в кружках. Это подтверждается как экспериментально, так и теоретически. Поскольку при нулевом магнитном поле эти $M I$-переходы ${ }^{87} \mathrm{Rb}$ имеют нулевую вероятность, следовательно, в магнитном поле происходит гигантское возрастание их вероятностей. Аналогичное заключение можно сделать и для $M I$-переходов ${ }^{85} \mathrm{Rb}$ с номерами $1-5$ в прямоугольниках: поскольку при нулевом магнитном

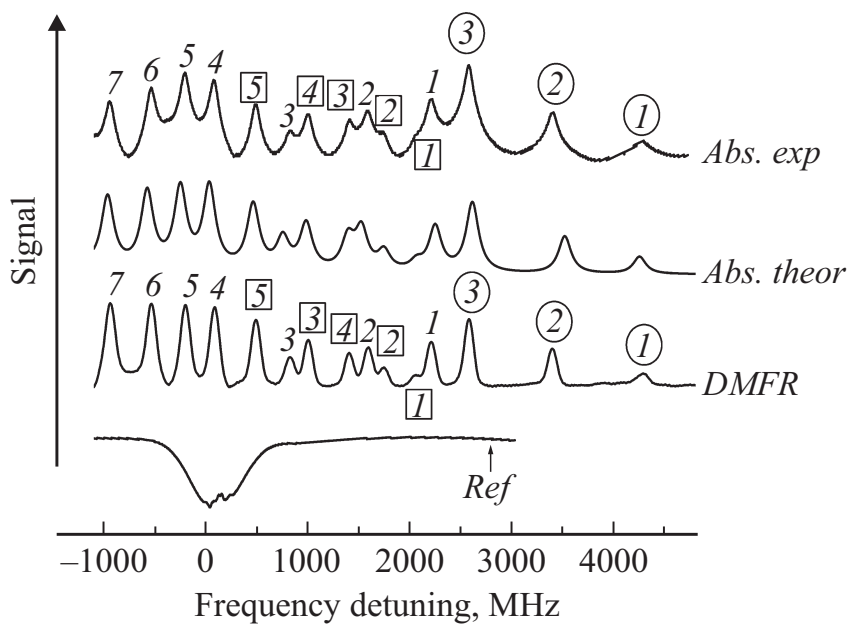

Рис. 4. Верхняя кривая (Abs. ехp) спектр поглощения НЯ, $B=1150 \mathrm{G}$; кривая Abs. Theor - расчетный спектр поглощения НЯ; кривая $D M F R$ - спектр сигнала производной кривой $M F R$. Обозначениями 1-3 в кружках и 1-5 в прямоугольниках показаны MI переходы атомов ${ }^{87} \mathrm{Rb}$ и ${ }^{85} \mathrm{Rb}$ соответственно. Нумерация переходов приведена на рис. 2, нижняя кривая $R e f$ - частотный репер. 
поле эти $M I$-переходы имеют нулевую вероятность, следовательно, в магнитном поле происходит гигантское возрастание их вероятностей. В работах [17-22] для количественного определения насколько сильно магнитное поле (для того или иного атома), была введена величина $B_{0}=A_{\mathrm{hfs}} / \mu_{\mathrm{B}}$, где $A_{\mathrm{hfs}}-$ магнитная дипольная константа для основного уровня атома, $\mu_{\mathrm{B}}-$ магнетон Бора [23]. Для атома ${ }^{85} \mathrm{Rb}$ величина $B_{0}\left({ }^{85} \mathrm{Rb}\right)=0.7 \mathrm{kG}$, а для атома ${ }^{87} \mathrm{Rb}$ величина $B_{0}\left({ }^{87} \mathrm{Rb}\right)=2.4 \mathrm{kG}$. Следует отметить, что при $B \gg B_{0}$ вероятности $M I$-переходов стремятся к нулю. Для атомов ${ }^{85} \mathrm{Rb}$ это поля $B>2 \mathrm{kG}$, а для атомов ${ }^{87} \mathrm{Rb}$ это поля $B>5 \mathrm{kG}$ [24].

\section{Пары атомов K}

В сильных магнитных полях, когда $B \gg B_{0}$ [24,25], имеет место разрыв связи полного углового момента электрона $\mathbf{J}$ и магнитного момента ядра I (режим Пашена-Бака на сверхтонкой структуре [17-22]), и поведение атомных переходов определяется проекциями $m_{J}$ и $m_{I}$. Дла атома ${ }^{39} \mathrm{~K}$ величина $B_{0}\left({ }^{39} \mathrm{~K}\right)=165 \mathrm{G}$ значительно меньше соответствуюших величин для атомов ${ }^{85} \mathrm{Rb}$ и ${ }^{87} \mathrm{Rb}$, поэтому разрыв связи $\mathbf{J}$ и $\mathbf{I}$ для ${ }^{39} \mathrm{~K}$ происходит при значительно меньших полях. По этой причине в случае использования атомов ${ }^{39} \mathrm{~K}$ такие важные особенности поведения атомных переходов как существенное изменение вероятностей атомных переходов и значительное уменьшение их числа (до фиксированного количества) с увеличением магнитного поля, могут быть легко получены при в 10-15 раз меньших магнитных полях. На рис. 5 приведены диаграммы атомных переходов в режиме Пашена-Бака на сверхтонкой структуре (HPB hyperfine Paschen-Back) в базе $m_{J}$ и $m_{I}$ для излучения с круговой поляризяцией $\sigma^{+}$для $D_{1}$-линии $(a)$ и для $D_{2}$ линии $(b)$. Правила отбора для переходов $\Delta m_{J}=+1$ и $\Delta m_{I}=0$. В случае излучения с круговой поляризацией $\sigma^{-}$правила отбора для переходов $\Delta m_{J}=-1$ и $\Delta m_{I}=0$. Эти переходы не показаны, чтобы не загромождать диаграмму. Регистрация спектров осуществлялась на высокочастотном крыле: это соответствует случаю применения излучения с круговой поляризяцией $\sigma^{+}$. Как видно из диаграммы на рис. $5, a$ в случае $D_{1}$-линии калия в режиме Пашена-Бака на сверхтонкой структуре в спектре для излучения с поляризацией $\sigma^{+}$должны присутствовать только четыре атомных перехода. При этом, как показано в работах $[21,22]$, их вероятности должны быть равны, а по частоте атомные линии 1-4 должны быть расположены практически эквидистантно. На рис. 6, $a$ кривые $D M F R$ показывают производную спектра сигнала $M F R$ на выходе из анализатора (повернут на $0.1 \mathrm{rad}$ ) при разных величинах магнитного поля $B$ : 320,365 ,425, 462 и 580 GG. Нумерация атомных линий 1-4 приведена на рис. 5,a. Отметим, что частотный интервал между атомными линиями на рис. 6 примерно $100 \mathrm{MHz}$, при допплеровской ширине атомных переходов $\sim 900 \mathrm{MHz}$ (при температуре $\sim 160^{\circ} \mathrm{C}$ ). Следовательно, метод DMFR работает очень эффективно. Как
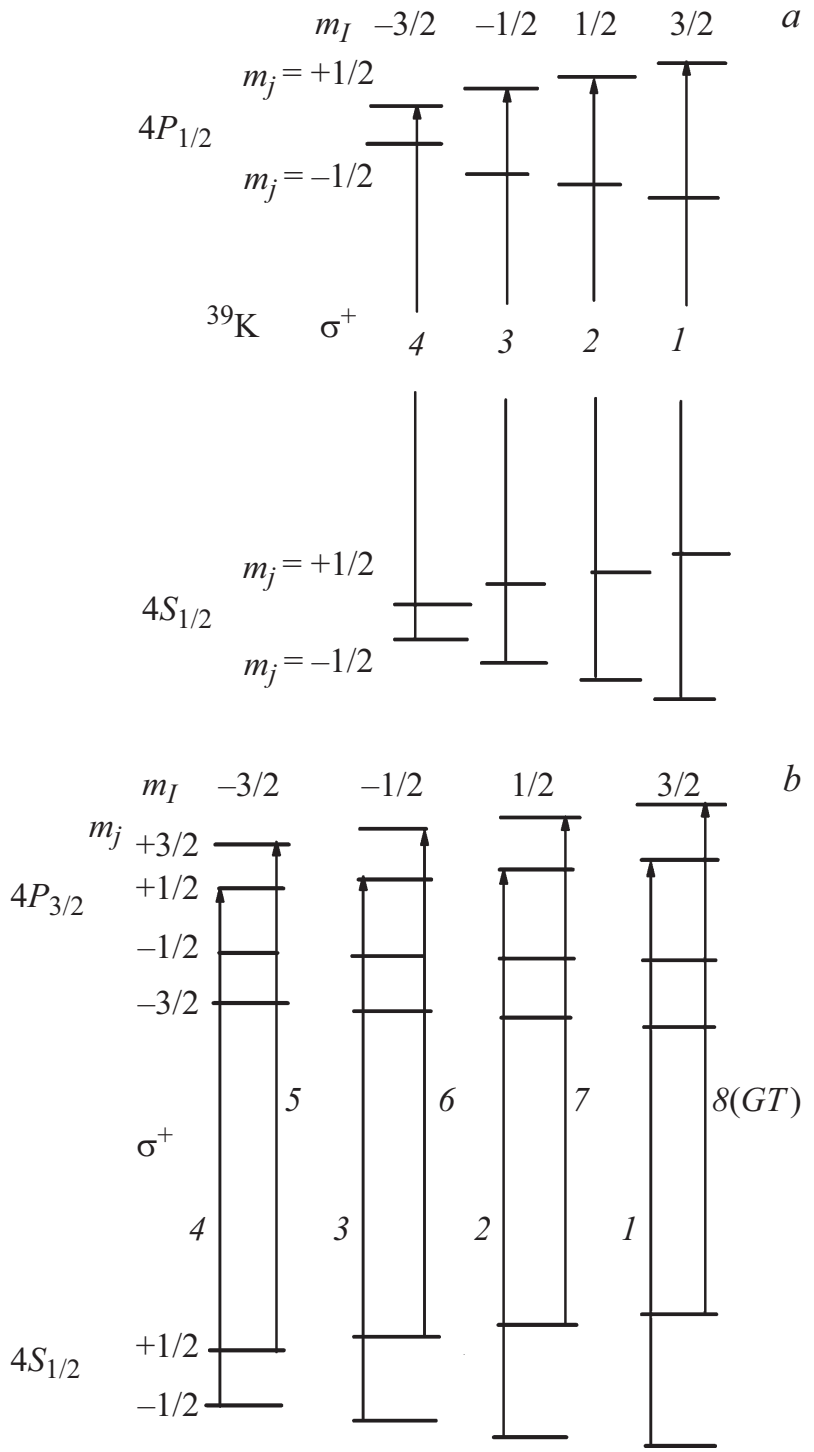

Pис. 5. (a) Диаграмма атомных переходов в режиме ПашенаБака на сверхтонкой структуре $(H P B)$ в базе $m_{J}$ и $m_{I}$ для излучения с поляризацией $\sigma^{+}$для $D_{1}$-линии и $(b)$ для $D_{2-}$ линии. В режиме $H P B$ в спектре присутствуют 4 и 8 атомных переходов соответственно для $D_{1}$ - и для $D_{2}$-линий. На диаграмме $(b)$ переход под номером $8-$ „направляющий“ переход (,guiding“ transitions, GT) [27].

видно по рис. $6, a$, режим Пашена-Бака на сверхтонкой структуре можно считать установившимся (по критериям, отмеченным выше) уже при $B>400 \mathrm{G}$. Нижняя кривая - частотный репер при нулевом магнитном поле.

Рассмотрим теперь случай $D_{2}$-линии атома ${ }^{39} \mathrm{~K}$. Как видно из диаграммы $(b)$, приведенной на рис. 5 , в режиме Пашена-Бака $(B \gg 165 \mathrm{G})$ для излучения с круговой поляризацией $\sigma^{+}$ожидается восемь атомных линий. На рис. 6, $b$ приведен экспериментальный спектр, и кривые $D M F R$ показывают производную спектра сигнала $M F R$ на выходе из анализатора при разных величинах 


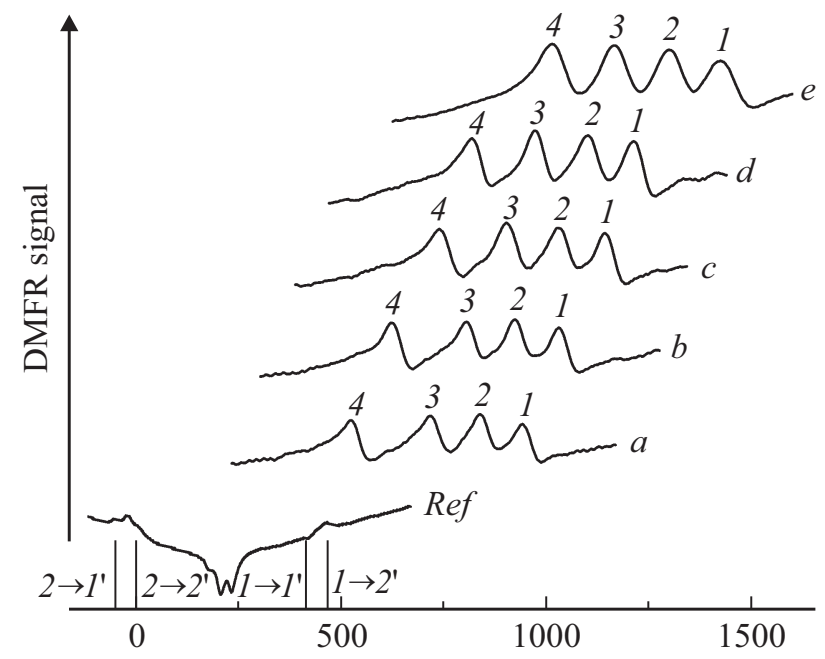

Frequency detuning, $\mathrm{MHz}$

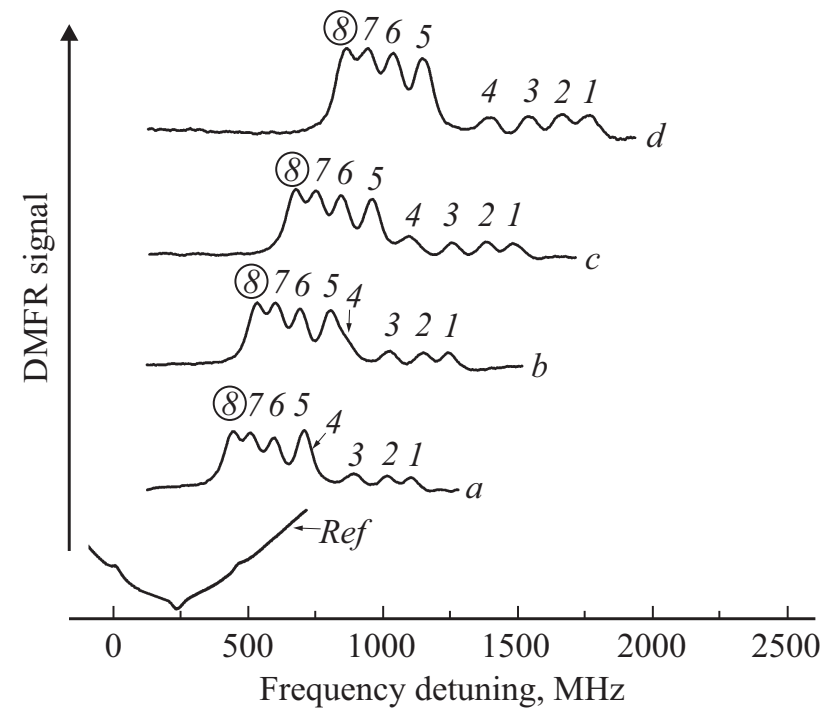

Рис. 6. (a) Кривые $D M F R$ - спектры ${ }^{39} \mathrm{~K}, D_{1}$-линия, при $B=320,365,425,462$ и $580 \mathrm{G}$. Нумерация переходов $1-4$ приведена на рис. $5, a$. Как видно, при $B>400 \mathrm{G}$ их вероятности равны, а по частоте переходы 1-4 расположены практически эквидистантно, что является проявлением режима $H P B$ для $D_{1}$-линии. Нижняя кривая $R e f-$ частотный репер. (b) Кривые $D M F R$ - спектры ${ }^{39} \mathrm{~K}, D_{2}$-линии, при $B=290,360,440$ и 590 G. Регистрируются две группы, в каждой по 4 атомных линии. Вероятности переходов в пределах группы равны между собой, а по частоте переходы расположены в группе почти эквидистантно, что является проявлением режима $H P B$ для $D_{2}$-линии. Нумерация переходов приведена на рис. $5, b$. Нижняя кривая $R e f$ - частотный репер.

магнитного поля $B: 290,360$,440 и 590 G. Спектральная ширина атомного перехода примерно $60 \mathrm{MHz}$ (полная ширина на полувысоте), что примерно в 15 раз уже допплеровской ширины $900 \mathrm{MHz}$. Как видно из рис. $6, b$, регистрируются две группы, в каждой из которых по четыре атомных линии. При этом, как показано в работе [26], вероятности переходов в пределах своей группы должны быть равны между собой, а по частоте
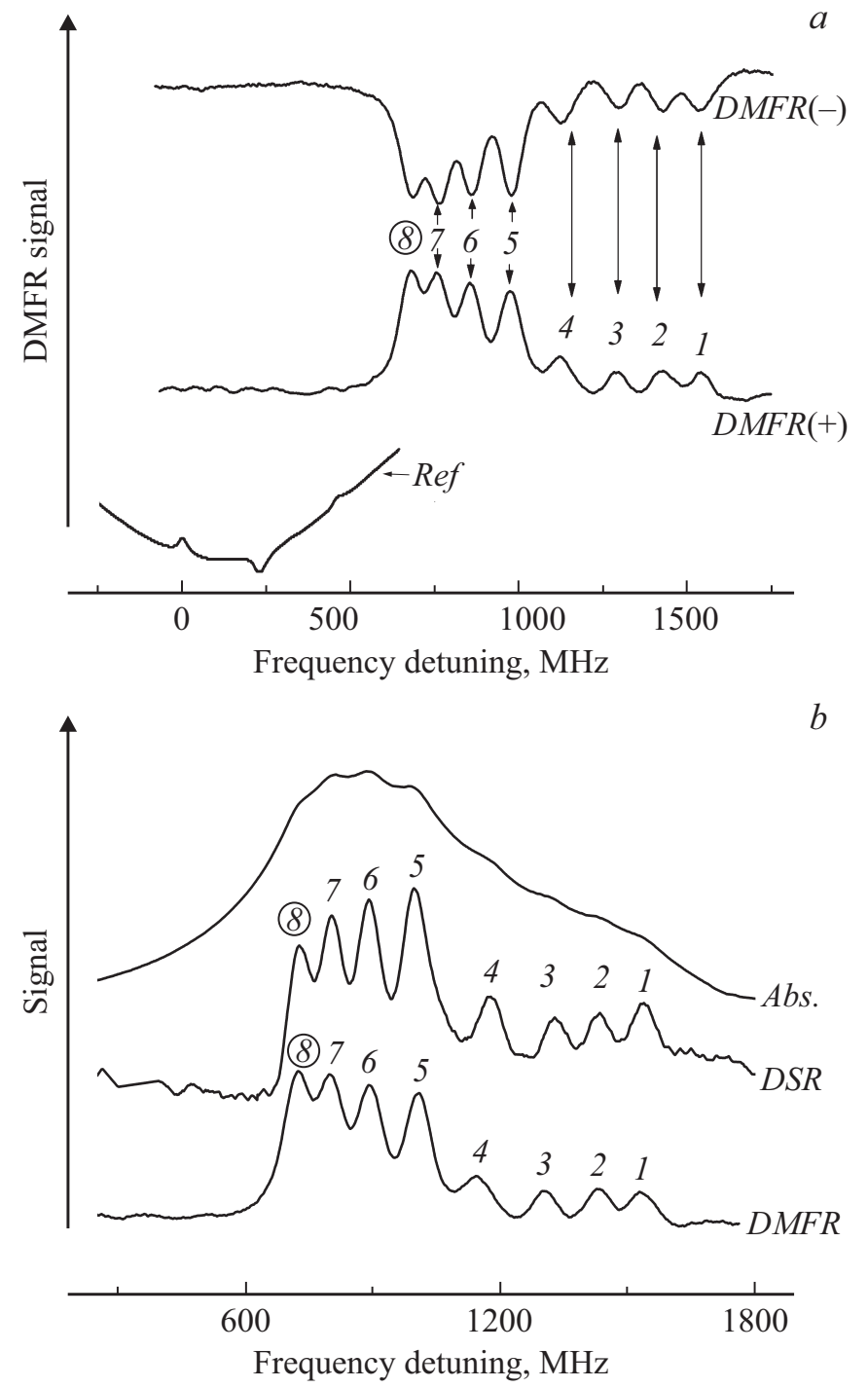

Pис. 7. (a) Кривые DMFR ( \pm ) показывают спектры ${ }^{39} \mathrm{~K}$, $D_{2}$-линии, при вращении анализатора по часовой стрелке на $+0.1 \mathrm{rad}$ или против часовой стрелки на $-0.1 \mathrm{rad}$ соответственно, $B=500 \mathrm{G}$, нижняя кривая $R e f-$ частотный репер; (b) верхняя кривая $A b s-$ спектр поглощения НЯ при $L \sim \lambda / 2$, $B=500 \mathrm{G}$, средняя кривая - спектр, полученный методом $S R$ (DSR - derivative of $S R)$, нижняя кривая - спектр, полученный методом DMFR. При примерно одинаковом спектральном разрешении $D M F R$ и $S R$ спектр $D M F R$ расположен на горизонтальной линии, что удобно для технических приложений.

атомные линии должны быть расположены практически эквидистантно. Нумерация атомных линий приведена на диаграмме $(b)$ на рис. 5. Особо отметим переход под номером 8 в кружке. Это так называемый „направляющий“ атомный переход (,guiding“transition, GT) при использовании излучения с круговой поляризацией $\sigma^{+}$(один такой $G T$-переход есть и при использовании излучения с поляризяцией $\sigma^{-}$) [26]. $G T$-переходы имеют наибольшую величину вероятности в своей группе, к которой стремятся вероятности всех атомных переходов 
в его группе в сильных магнитных полях. Величина частотного наклона $G T$-перехода (определяет частотный сдвиг перехода в зависимости от $B$ ) составляет $s=+1.4 \mathrm{MHz} / \mathrm{G}$. В сильных магнитных полях к этой величине стремятся частотные наклоны всех атомных переходов в его группе [26,27]. Как видно по рис. 6, b, режим Пашена-Бака на сверхтонкой структуре можно считать установившимся уже при $B>400 \mathrm{G}$. Отметим, что частотный интервал между атомными линиями на pис. $6, b \sim 100 \mathrm{MHz}$ при допплеровской ширине атомных переходов $\sim 900 \mathrm{MHz}$. Следовательно, метод $D M F R$ работает очень эффективно и для $D_{2}$-линии атома ${ }^{39} \mathrm{~K}$. Отметим, что на рис. 6 приведено высокочастотное крыло спектра, возбуждаемое излучением $\sigma^{+}$-поляризацией, аналогичный спектр формируется и на низкочастотном крыле при использовании излучения с $\sigma^{-}$-поляризацией. Заметим, что вследствие малости величины $B_{0}$ для атома ${ }^{39} \mathrm{~K}$ вероятности $M I$ переходов для отмеченных на рис. $6, b$ магнитных полей быстро уменьшаются и практически не регистрируются в сильных магнитных полях. Интересно отметить следующее. На рис. 7, $a$ приведены спектры, полученные методом $D M F R( \pm)$ при вращении анализатора по часовой стрелке $(+)$ и против часовой стрелки $(-)$ на ту же величину $( \pm 0.1 \mathrm{rad})$ соответственно. Как видно по рис. 7, $a$, частотные интервалы, а также относительные амплитуды в пределах своей группы при этом не изменяются. Нижняя кривая - частотный репер при нулевом магнитном поле.

Важно отметить, что до последнего времени считалось, что лучшее спектральное разрешение атомных линий достигается в случае применения процесса селективного отражения $(S R)$ от наноячейки при толщине $L \sim \lambda / 2$, что позволяет спектральное разделение большого количества атомных переходов, которые формируются в сильных магнитных полях [11]. Поэтому целесообразно сравнить метод DMFR и метод $D S R$ (derivative of $S R$ ) [11]. На рис. 7,b приведены спектры, полученные методом $D M F R$, методом $S R$ и методом регистрации спектра поглощения наноячейки при толщине $L \sim \lambda / 2$. Как видно из рисунка, оба метода $D M F R$ и $D S R$ обеспечивают значительно лучшее спектральное разрешение, чем метод регистрации спектра поглощения наноячейки. При примерно одинаковом спектральном разрешении методов DMFR и DSR к преимуществу первого можно отнести то, что спектр формируется практически на горизонтальной линии, что удобно при необходимости разложения огибающей на исходные составляющие компоненты. К преимуществу метода $D M F R$ можно отнести и то, что регистрируется спектр проходяшего через наноячейку излучения, в то время как в случае процесса $S R$ регистрируется отраженный от наноячейки сигнал. В частности, при использовании постоянного магнита с малым отверстием для лазерного излучения для метода $D M F R$ такое маленькое отверстие приемлемо, в то время как для реализации процесса SR оно создает технические трудности.

\section{Заключение}

Разработан и применен модифицированный метод фарадеевского вращения $(D M F R)$ с использованием наноячейки, заполненной парами атомов $\mathrm{Rb}$ и $\mathrm{K}$. Суть модифицированного метода состоит в следующем: выходной поляризатор (анализатор) помещается в держатель, который позволяет осушествлять его плавное вращение вокруг оси как по часовой стрелке, так и против часовой стрелки. Угол, на который поворачивался анализатор (относительно скрещенного положения) составлял $0.1 \mathrm{rad}$. В этом случае на выходе анализатора регистрируется дисперсионный спектр. Используемый осциллограф Siglent позволяет в реальном времени формировать производную кривой $M F R$. Формируемые атомные линии имеют спектральную ширину $50-70 \mathrm{MHz}$, что в $1.5-2$ раза уже, чем спектральная ширина, полученная методом фарадеевского вращения в наноячейке. В магнитных полях 100-1200 G спектрально разрешены и идентифицированы все атомные линии ${ }^{85} \mathrm{Rb},{ }^{87} \mathrm{Rb}$ и ${ }^{39} \mathrm{~K}$. В случае $D_{2}$ линии $\mathrm{Rb}$ экспериментально и теоретически показано, что при значениях магнитного поля в интервале $600-1300 \mathrm{G}$ вероятности магнито-индуцированных переходов ${ }^{87} \mathrm{Rb}$, $F_{g}=1 \rightarrow F_{e}=3$ и ${ }^{85} \mathrm{Rb}, F_{g}=2 \rightarrow F_{e}=4$ (которые запрещены по правилам отбора при $B=0$ ) могут превосходить вероятности разрешенных переходов. В случае атомов ${ }^{39} \mathrm{~K}, D_{1}$-линии при значениях магнитного поля в интервале 300-600 G на высокочастотном крыле с помощью метода $D M F R$ регистрируются четыре атомные линии примерно равной амплитуды с частотным интервалом между линиями $\sim 100 \mathrm{MHz}$. В случае атомов ${ }^{39} \mathrm{~K}, D_{2}$-линии при значениях магнитного поля в интервале $300-600 \mathrm{G}$ на высокочастотном крыле с помощью метода $D M F R$ регистрируются две группы атомных переходов по четыре перехода в каждой группе. Показано, что в зависимости от направления врашения анализатора $( \pm 0.1 \mathrm{rad})$ знак амплитуды сигнала $D M F R$ изменяется с положительного на отрицательный (при этом все остальные характеристики спектров остаются одинаковыми). Проведено сравнение спектров, формируемых методом селективного отражения и методом $D M F R$, и показано, что реализуемое спектральное разрешение обоих методов примерно одинаково. Однако в случае применения магнитных полей метод $D M F R$ имеет некоторые преимущества.

Авторы благодарят А. Папояна, А. Тонояна и Г. Ахумяна за полезные обсуждения. Работа А. Саргсяна, А. Амиряна и Д. Саркисяна выполнена в рамках ГКН MOH PA (проект № SCS 18T-1C018)". Авторы также благодарят Армянский национальный фонд науки и образования (грант ANSEF Opt 4732) за финансовую поддержку. А.А. благодарит AGBU France и Philippossian \& 
Pilossian Foundation в Женеве, а также FAST (Foundation of Armenian Science and Technology). Работа T.A. Вартаняна выполнена в рамках госзадания № 3.4903.2017/6.7.

\section{Список литературы}

[1] Budker D., Gawlik W., Kimball D. et al. // Rev. Mod. Phys. 2002. V. 74. P. 1153.

[2] Yashchuk V.V., Budker D., Gawlik W. et al. // Phys. Rev. Lett. 2003. V. 90. P. 253001.

[3] Auzinsh M., Budker D., Rochester M. Optically Polarized Atoms: Understanding Light-Atom Interactions. Oxford University Press, 2010.

[4] Wang Y., Zhang X., Wang D., Tao Z., Zhuang W., Chen J. // Opt. Express. 2012. V. 20. P. 25817.

[5] Zielińska J.A., Beduini F.A., Godbout N., Mitchell M.W. // Opt. Lett. 2012. V. 37. P. 524.

[6] Zentile M.A., Whiting D.J., Keaveney J., Adams Ch.S., Hughes I.G. // Opt. Lett. 2015. V. 40. P. 2000.

[7] Zentile M.A, Andrews R., Weller L., Knappe S., Adams Ch.S., Hughes I.G. // J. Phys. B. 2014. V. 47. P. 075005.

[8] Саргсян А., Пашаян-Леруа Е., Леруа К., Малакян Ю., Саркисян Д. // Письма в ЖЭТФ. 2015. Т. 102. С. 549

[9] Саргсян А., Пашаян-Леруа Е., Леруа К., Саркисян Д. // ЖЭТФ. 2016. Т. 150. С. 461.

[10] Саргсян А., Амирян А., Карталева С., Саркисян Д. // ЖЭТФ. 2017. Т. 152. С. 54.

[11] Саргсян А., Клингер Э., Пашаян-Леруа Е., Леруа К., Папоян А., Саркисян Д. // Письма в ЖЭТФ. 2016. Т. 104. C. 222.

[12] Vartanyan T.A., Lin D.L. // Phys. Rev. A. 1995. V. 51. P. 1959.

[13] Sargsyan A., Tonoyan A., Hakhumyan G., Leroy C., Pashayan-Leroy Y., Sarkisyan D. // Europhys. Lett. 2015. V. 110. P. 23001.

[14] Саргсян А., Амирян А., Леруа К., Вартанян Т.А., Саркисян Д. // Опт. и спектр. 2017. Т. 123. № 2. С. 113.

[15] Peyrot T., Sortais Y.R.P., Browaeys A., Sargsyan A., Sarkisyan D., Keaveney J., Hughes I.G., Adams C.S. // Phys. Rev. Lett. 2018. V. 120. P. 243401.

[16] Demtröder W. // Laser Spectroscopy: Basic Concepts and Instrumentation. Springer-Verlag, 2004.

[17] Tremblay P., Michaud A., Levesque M., Th'eriault S., Breton M., Beaubien J., Cyr N. // Phys. Rev. A. 1990. V. 42. P. 2766.

[18] Sargsyan A., Tonoyan A., Hakhumyan G., Papoyan A., Mariotti E., Sarkisyan D. // Laser Phys. Lett. 2014. V. 11. P. 055701.

[19] Sargsyan A., Klinger E., Hakhumyan G., Tonoyan A., Papoyan A., Leroy C., Sarkisyan D. // J. Opt. Soc. Am. B. 2017. V. 34. P. 776.

[20] Саргсян А., Тоноян А., Ахумян Г., Саркисян Д. // Письма в ЖЭТФ. 2017. Т. 106. С. 669.

[21] Tonoyan A., Sargsyan A., Klinger E. et al. // EuroPhys. Lett. 2018. V. 121. P. 53001.

[22] Саргсян А., Амирян А., Вартанян Т.А., Саркисян Д. // Опт. и спектр. 2016. Т. 121. № 5. С. 848.

[23] Zentile M., Keaveney J., Weller L., Whiting D.J., Adams C.S., Hughes I.G. // Comput. Phys. Commun. 2015. V. 189. P. 162.

[24] Sargsyan A., Hakhumyan G., Leroy C., Pashayan-Leroy Y., Papoyan A., Sarkisyan D. // Opt. Lett. 2012. V. 37. P. 1379.
[25] Olsen B.A., Patton B., Jau Y.Y., Happer W. // Phys. Rev. A. 2011. V. 84. P. 063410.

[26] Sargsyan A., Klinger E., Tonoyan A. et al. // J. Phys. B. 2018. V. 51. P. 145001.

[27] Саргсян А., Ахумян Г., Папоян А., Саркисян Д. // Письма в ЖЭТФ. 2015. Т. 101. С. 330. 